Research Article

\title{
Computed Tomography Image Segmentation Using Edge Correction Algorithm for Refractory Mycoplasma Pneumonia in Children
}

\author{
Lijuan Wu ${ }^{1}$, ${ }^{1}$ Jianwei Ji $\mathbb{D}^{1},{ }^{1}$ Shiyong Zhao $\mathbb{D}^{2},{ }^{2}$ and Jiaolei Chen $\mathbb{D D}^{3}$ \\ ${ }^{1}$ Department of Pediatrics, Yiwu Central Hospital, Yiwu 322000, China \\ ${ }^{2}$ Department of Pediatric Internal Medicine, Hangzhou Children's Hospital, Hangzhou 310014, China \\ ${ }^{3}$ Department of Neonatology, Yiwu Central Hospital, Yiwu 322000, China
}

Correspondence should be addressed to Jianwei Ji; 20200120876@nxmu.edu.cn

Received 5 June 2021; Revised 28 June 2021; Accepted 12 July 2021; Published 20 July 2021

Academic Editor: Gustavo Ramirez

Copyright (c) 2021 Lijuan Wu et al. This is an open access article distributed under the Creative Commons Attribution License, which permits unrestricted use, distribution, and reproduction in any medium, provided the original work is properly cited.

Objective. It is to study the application of edge correction algorithm (ECA) in computed tomography (CT) medical image segmentation, explore its guiding significance in the analysis of clinical characteristics of children with refractory mycoplasma pneumoniae (RMPP), and discuss the therapeutic value of fiberoptic bronchoscopy bronchoalveolar lavage (BAL) for RMPP. Methods. The accuracy of ECA in CT medical image segmentation of children with RMPP was compared with that of the watershed segmentation algorithm (WSA) and swarm intelligence optimization algorithm (SIOA). The clinical characteristics and the imaging characteristics of 80 children with RMPP admitted to hospital from January 2018 to January 2020 were retrospectively analyzed based on the ECA. All children were divided into a lavage group (BAL group, $n=69$ ) and a nonlavage group (non-BAL group, $n=11$ ) according to whether fiberoptic bronchoscopy and BAL were performed. Bronchoscopy was adopted to analyze the cytological characteristics of BAL fluid (BALF) in children, and the recovery rate and the total effective rate of the two groups of children were observed and compared. Results. The overall accuracies (OAs) of the three ECAs (Roberts operator (RO), Sobel operator (SO), and Prewitt operator (PO)) were higher than that of WSA and SIOA, their false negative rate (FNR) and false positive rate (FPR) were small, and their denoising performance was superior to that of WSA and SIOA. The main clinical manifestations of all children were high fever, irritating dry cough, and few early signs. The results of chest CT examination were mainly manifested as patchy or large-scale consolidation, two lung mesh or small nodular shadows, and atelectasis. 69 cases with fiberoptic bronchoscopy showed swelling and congestion of the bronchial mucosa at the lesion site with visible viscous secretions, which was consistent with the imaging changes. The total number of cells in the BALF of children increased $(P<0.05)$, which mainly represented the increase of neutrophils $(P<0.05)$. The recovery rate of children with lavage $(81.16 \%)$ was higher dramatically than that of the nonlavage group (45.45\%). Conclusion. The ECA had good accuracy and denoising performance in lung CT image segmentation. The clinical characteristics, imaging characteristics, and cytological components of children had changed when they suffered from the RMPP, and fiberoptic bronchoscopy lavage had a therapeutic effect on it.

\section{Introduction}

Mycoplasma pneumonia (MP) is one of the most common pathogens that causes community acquired pneumonia (CAP) in children [1]. Studies have shown that MP may account for $40 \%$ of CAP cases, and $18 \%$ of children have to be treated with hospitalization. Although mycoplasma pneumoniae pneumonia (MPP) is generally considered to be a self-limiting disease, it can sometimes cause various pulmonary and extrapulmonary complications, such as bronchiolitis obliterans, necrotizing pneumonia, encephalitis, arthritis, pericarditis, hemolytic anemia, and the development of severe life-threatening pneumonia [2]. For children, macrolides are the antibiotics of choice for MP 
infection. However, macrolide antibiotic treatment lasts for 7 days or longer, but there are still some cases showing clinical and radiological deterioration, which is defined as RMPP [3]. Therefore, it is extremely important for clinicians to identify RMPP as soon as possible and promptly conduct reasonable treatment. RMPP lacks an early specific diagnosis method and currently mainly depends on clinical and imaging examination results, but these examination results often lack specificity. In recent years, the application of fiberoptic bronchoscopy in pediatrics has been developed, which has hugely improved the diagnosis of pediatric respiratory diseases. At present, fiberoptic bronchoscopy has become one of the important methods for the diagnosis of RMPP. It is generally found that RMPP has certain characteristics under the microscope [4].

The image segmentation algorithm is based on the discontinuity and similarity properties shown by the gray value. The area where the gray value of the pixel is not continuous is considered as the edge of the image; the gray value of the pixels around the edge will change sharply, based on which the target area can be divided [5]. In the image, the neighborhoods of the same area often have similar or the same gray value, but in medical images, there are often uneven gray levels, so the target area is accurately segmented for image feature extraction and recognition analysis of the later medical diagnosis system. It has a great promotion effect [6]. The current image segmentation algorithms are mainly divided into regionbased image segmentation, edge-based image segmentation, and specific theory-based image segmentation [7]. Edge-based image segmentation cannot effectively segment the image because the calculation of its derivative is extremely sensitive to noise, so it is necessary to reduce the impact of noise during image preprocessing [8]. Thus, it is more widely used in medical image segmentation. Research studies show that the ECA has more advantages than the WSA in magnetic resonance imaging (MRI) image segmentation, and the accuracy rate is $5 \%$ higher than that of the WSA. However, there are few research studies on the application of ECA in CT image segmentation. Thus, this study analyzes the advantages of the ECA in segmentation of chest CT images of children with RMPP.

In this study, three different differential edge operators were utilized to preprocess the image, and the accuracy and denoising performance in image segmentation were compared and analyzed. At the same time, it summarized and analyzed the clinical characteristics, imaging examination results, and differences of fiberoptic bronchoscopy in children with RMPP, compared and analyzed the performance before and after the treatment of fiberoptic bronchoscopy lavage in children, and explored its therapeutic value for RMPP, so as to provide positive effects for early diagnosis and treatment of RMPP.

\section{Materials and Methods}

2.1. CT Image Based on Edge Correction Algorithm. In the medical images, the derivative operator was extremely sensitive to weak changes in pixel values, so the edge area of the image could be detected by the derivative operator, and the edge could be determined based on the prediction threshold. It was supposed that the image was $f(x, y)$. The operators calculated according to the one-dimensional partial derivative were $\partial f / \partial x$ and $\partial f / \partial y$, respectively, which represented the value changes along the $x$-axis and $y$-axis. In equation (1), it was supposed that the rate of change of the $Z$-axis was the superposition of the $x$-axis and $y$-axis, and the differential of the continuous function was given in equation (2). In the digital images, differential operation and derivative operation could be converted into difference operation, and equations (3)-(5) represented the forward and backward difference and direction difference, respectively:

$$
\begin{aligned}
\frac{\partial f}{\partial z} & =\frac{\partial f}{\partial x} \cos z+\frac{\partial f}{\partial y} \sin z \\
d f & =\frac{\partial f}{\partial x} d x+\frac{\partial f}{\partial y} d y, \\
\Delta f(i, j) & =f(i, j)-f(i-1, j-1), \\
\Delta f(i, j) & =f(i, j)-f(i+1, j+1), \\
\Delta_{z} f(i, j) & =\Delta_{x} f(i, j) \cos z-\Delta_{j} f(i, j) \sin z .
\end{aligned}
$$

The derivative of the function $f(x, y)$ could obtain the maximum value at a point; then, its direction was represented with equation (6), its modulus value could be calculated with equation (7), and the modulus determination function gradient was indicated by equation (8); then, the operator of a gradient modulus could be defined as equation (9). In the following equations, $\partial$ represents the partial derivative, $\nabla$ represents the gradient, and $G$ refers to the gradient operator:

$$
\begin{gathered}
\arctan \left[\frac{\partial f / \partial y}{\partial y / \partial x}\right], \\
\sqrt{\left(\frac{\partial f}{\partial x}\right)^{2}+\left(\frac{\partial f}{\partial y}\right)^{2},} \\
\nabla f(x, y)=\left(\frac{\partial f}{\partial x}, \frac{\partial f}{\partial y}\right)^{\prime}, \\
G[f(x, y)]=\sqrt{\left(\frac{\partial f}{\partial x}\right)^{2}+\left(\frac{\partial f}{\partial y}\right)^{2}} .
\end{gathered}
$$

The gradient operator could accurately calculate the boundary in all directions, and the normal direction boundary could be calculated by equation (6). When the gradient corresponding to the image was calculated, equation (10) was applicable, or the gradient modulus could be substituted with an approximate gradient value, as shown in equation (11) and (12): 


$$
\begin{aligned}
& G[f(i, j)]=\left[\Delta_{x} f(i, j)^{2}+\Delta_{y} f(i, j)^{2}\right]^{1 / 2}, \\
& G[f(i, j)]=\left|\Delta_{x} f(i, j)\right|+\left|\Delta_{y} f(i, j)\right|, \\
& G[f(i, j)]=\max \left[\left|\Delta_{x} f(i, j)\right|+\left|\Delta_{y} f(i, j)\right|\right] .
\end{aligned}
$$

When the approximate gradient value had an impact on accuracy of the gradient direction, the actual algorithm had to be determined based on the actual image and the magnitude of the gradient information error. Figure 1 shows the comparison on the effects of the RO, PO, and SO in lung image segmentation.

The OAs of these three edge operators were classic WAS and SIOA in CT medical image segmentation of children with RMPP.

2.2. Research Objects and Grouping. 80 children with RMPP admitted to the hospital from January 2018 to January 2020 were analyzed retrospectively, including 47 male and 33 female patients with an average age of $5.01 \pm 2.31$ years old. The course before admission was 2-36 days, so the median was 7 days. The inclusion criteria were defined as below: all children met the diagnostic criteria for pneumonia in the seventh edition of Practical Pediatrics, children with double serum MP antibody titer increased by more than 4 times within 4 weeks of onset, and children whose family members informed and signed the informed consent forms. On the contrary, the exclusion criteria could be determined as follows: children with other severe organic diseases, children with pneumonia caused by other pathogenic bacteria, and children whose family members did not agree to participate in this study. This trial had been approved by Ethics Committee of hospital.

According to whether fiberoptic bronchoscopy BAL was performed, all children were grouped into a lavage group (BAL group, $n=69$ ) and a nonlavage group (non-BAL group, $n=11$ ). Among them, there were 41 males and 28 females in the BAL group, aged 0-14 years old, with an average age of $5.16 \pm 2.62$ years old. The course of disease was 3-18 days on admission, with a median of 6 days. In the nonBAL group, there were 6 males and 5 females, aged 0-14 years old, with the average age of $4.83 \pm 1.76$ years old. In addition, the course of disease was 2-36 days on admission with the median of 8 days. There was no statistical difference in the general data of the two groups of children in terms of gender, age, and course of disease at the time of admission $(P>0.05)$.

\subsection{Observation Indicators}

2.3.1. Clinical Symptoms. Fever, diarrhea, vomiting, coughing, and other signs were collected for all children.

2.3.2. Imaging Data. It mainly referred to the chest CT images of children, and the ECA was adopted to resegment the images to reacquire the characteristics of CT images.
2.3.3. Fiberoptic Bronchoscopy Indicators. After local anesthesia, the child was examined with a fiberoptic bronchoscope manufactured by Olympus, Japan. After the fiberoptic bronchoscope entered the airway, the diseased part was explored and lavaged, other parts and the contralateral bronchi were inspected, and the morphological changes of RMPP were observed and recorded. In addition, the BALF of the children was collected. After the BALF specimen was filtered, it was centrifuged at $4^{\circ} \mathrm{C}$ and $1500 \mathrm{r} / \mathrm{min}$ for 10 minutes, and the sediment was collected for cytological analysis. The result was expressed as $\times 10^{6} / \mathrm{mL}$.

2.3.4. Treatment Effect. All children were treated with the same treatment plan for 2 weeks, and the recovery rate and total effective rate of the two groups of children were observed and compared.

2.4. Statistical Analysis. All data were analyzed using SPSS22.0 software. The measurement data were expressed as mean \pm standard deviation, and the $t$-test was employed for comparison between groups. The enumeration data was given by the number of cases (percentage), and the chisquare test was adopted for the comparison between the two groups. $P<0.05$ means the difference was observably and statistically meaningful.

\section{Results}

3.1. Comparison of Lung CT Image Segmentation Parameters with Different Algorithms. The analysis results of lung CT image segmentation parameters with different algorithms were shown in Figures 2 and 3. The OA, FPR, and FNR of the RO were $0.935,0.0427$, and 0.0465 , respectively. The OA, FPR, and FNR of the SO were 0.0.931, 0.0453, and 0.0472, respectively; these three indicators of the PO were 0.925 , 0.0495 , and 0.0478 , respectively, those of WSA were 0.855 , 0.0892 , and 0.0875 , respectively, and those of OA, FPR, and FNR of SIOA were $0.847,0.1140$, and 0.0752 , respectively. Thus, the OAs of RO, SO, and PO were higher than that of WSA and SIOA, and the FPRs and FNRs of the three operators were lower in contrast to the WSA and SIOA.

\subsection{Comparison of Lung CT Image Segmentation in Denoising} Performance of Different Algorithms. The lung CT image segmentations with different algorithms were compared in terms of denoising performance, and the results were shown in Figure 4. The peak signal-to-noise ratio (PSNR) and the edge preservation index (E) of RO were 27.916 and 0.945 , respectively; the PSNR and E of SO were 27.855 and 0.95 , respectively; the PSNR and E of PO were 27.796 and 0.944 ; the PSNRs of WSA and SIOA were 26.554 and 26.871, respectively; and the Es of WSA and SIOA were 0.955 and 0.945 , respectively. Figure 4 illustrates that the PSNRs of the three edge algorithms (PO, SO, and RO) were higher than that of WSA and SIOA; the E value of WSA algorithm was the highest, followed by SO; the E values of RO and PO were close to that of SIOA. 


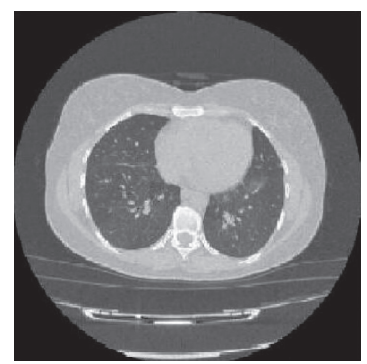

(a)

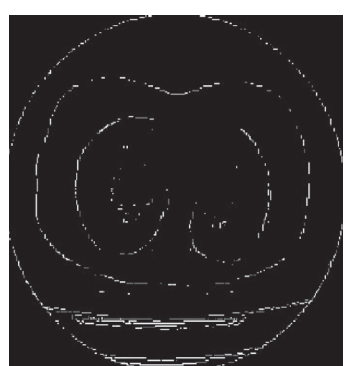

(b)

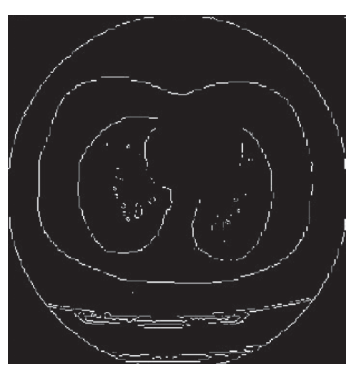

(c)

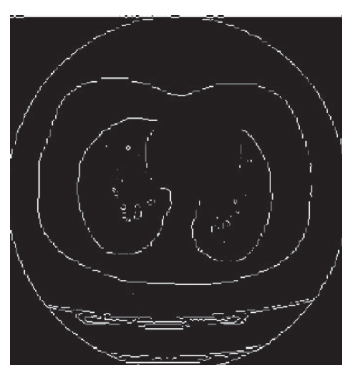

(d)

Figure 1: The effect diagram of different edge operators in lung image segmentation. (a), (b), (c), and (d) show the effects of the original image, $\mathrm{RO}$, SO, and $\mathrm{PO}$, respectively.

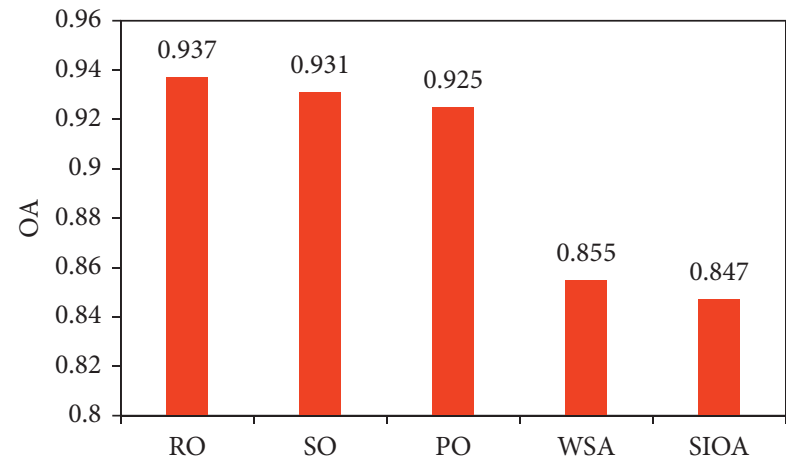

Figure 2: Comparison on overall accuracy of lung CT image segmentation with different algorithms.

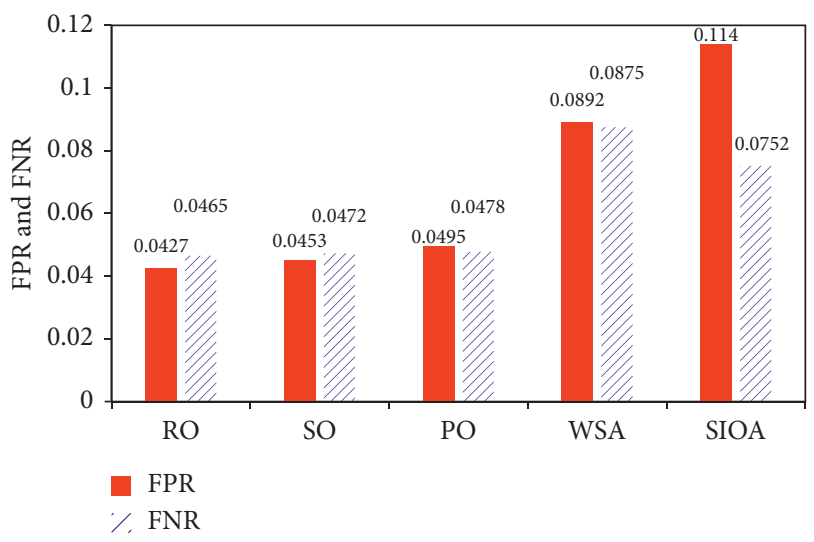

FIGURE 3: Comparison on false positive rate and false negative rate of lung CT image segmentation with different algorithms.

\subsection{Analysis of Clinical Symptoms and Signs in All Children.} The clinical symptoms such as fever, fever duration, early lung signs, and cough of all 80 children were observed and analyzed, and the results were shown in Figures 5 and 6. There were 25 children $(31.25 \%)$ with fever at $38.0-39.0^{\circ} \mathrm{C}$, 47 cases $(58.75 \%)$ at $>39.0^{\circ} \mathrm{C}$, and 8 cases $(10 \%)$ in other cases. For the fever duration, there were 20 cases (25\%) for less than 7 days, 58 cases (72.5\%) for 7-14 days, and 2 cases (2.5\%) for longer than 14 days and less than 20 days. In children with early lung signs (within 7 days of onset), there were 57 cases $(71.25 \%)$ with positive signs and 23 cases

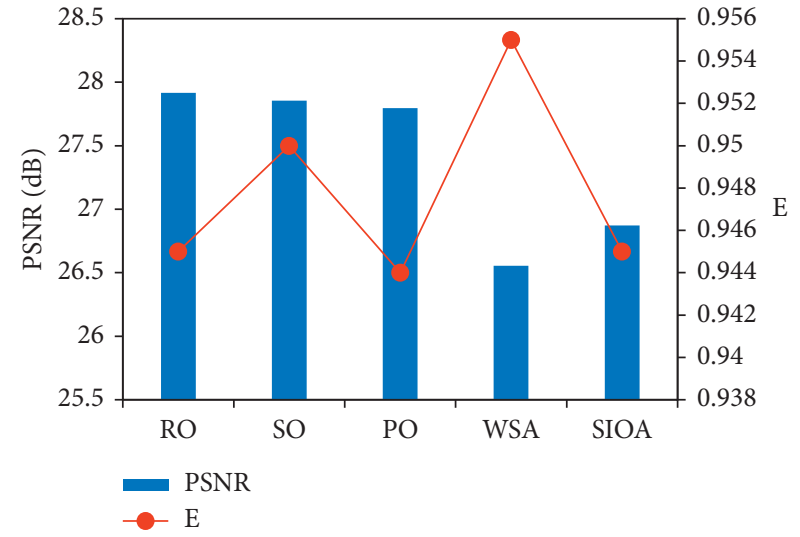

FIgUre 4: The lung CT image segmentations with different algorithms were compared in terms of denoising performance.

(28.75\%) with negative signs. All children suffered from cough, including 75 cases (93.75\%) of dry cough, 6 cases (7.5\%) of wheezing, 6 cases $(7.5 \%)$ of cough with chest pain, 4 cases (5\%) with bloody sputum, 24 cases (30\%) with stuffy nose, and 38 cases (47.5\%) with shortness of breath. Therefore, children with RMPP mainly suffered from high fever and irritating dry cough as their main symptoms. Most of the fevers had a course of 7-14 days with fewer early signs.

3.4. Imaging Analysis of All Children. The chest CT imaging indications of all 80 children were analyzed, as shown in Figure 7 . There were 52 children $(65 \%)$ with patchy or large consolidation, 16 children (20\%) with bronchial inflation phase, 22 children (27.5\%) with thickened bronchial wall, 16 children (20\%) with small tree bud-like sign, 32 children (40\%) with reticulated and nodular shadows in both lungs, 19 children (23.75\%) with focal uneven inflation of the lung field, 3 children (3.75\%) with spherical lesions, 33 children (41.25\%) with atelectasis and hilar or mediastinal lymph nodes, 8 cases $(10 \%)$ with swelling, 14 cases (17.5\%) with pleural effusion, 3 cases (3.75\%) with pneumothorax, and 13 cases $(16.25 \%)$ with migratory disease. It suggested that the chest CT imaging of children with RMPP was mainly characterized by patchy or large-scale consolidation, reticulated or nodular shadows of two lungs, and atelectasis. 


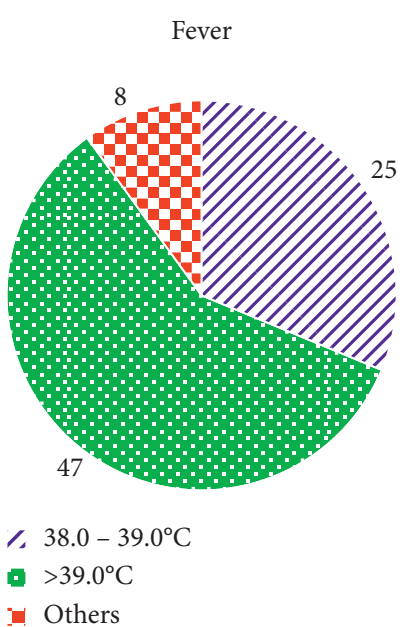

(a)

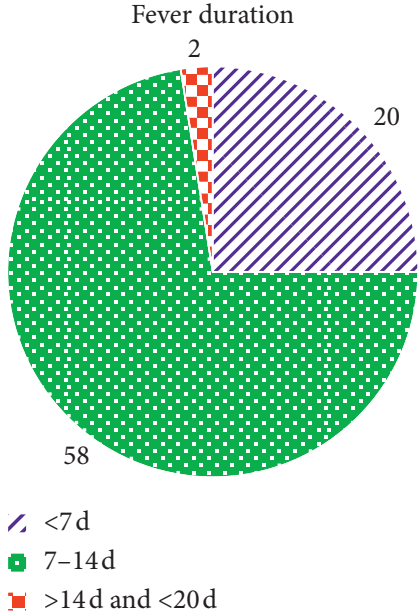

(b)

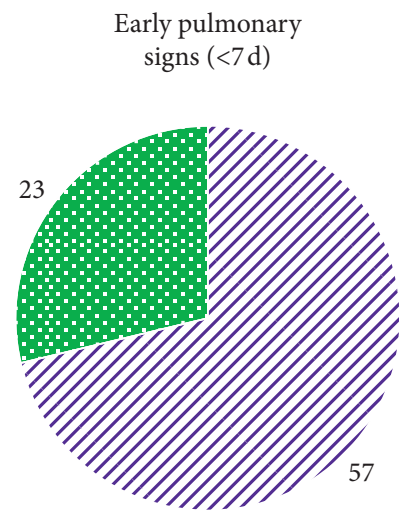

1. Positive signs

- Negative signs

(c)

Figure 5: Fever, fever duration, and pulmonary signs of the patients.

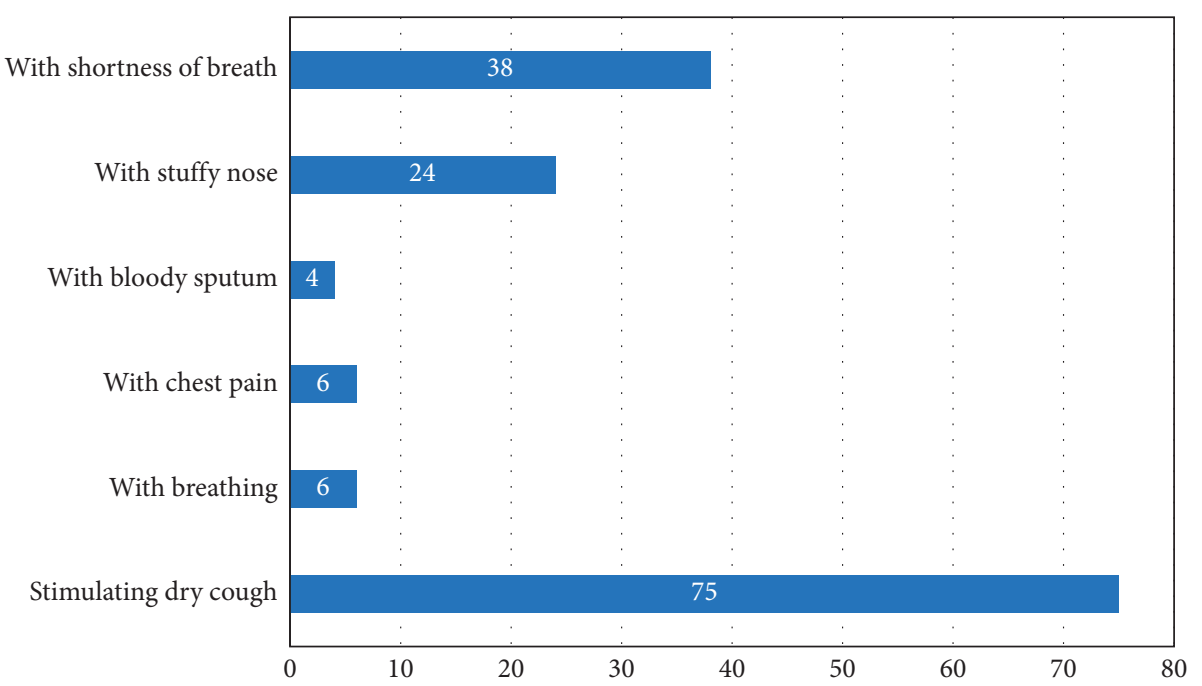

Figure 6: Analysis of cough symptoms in children.

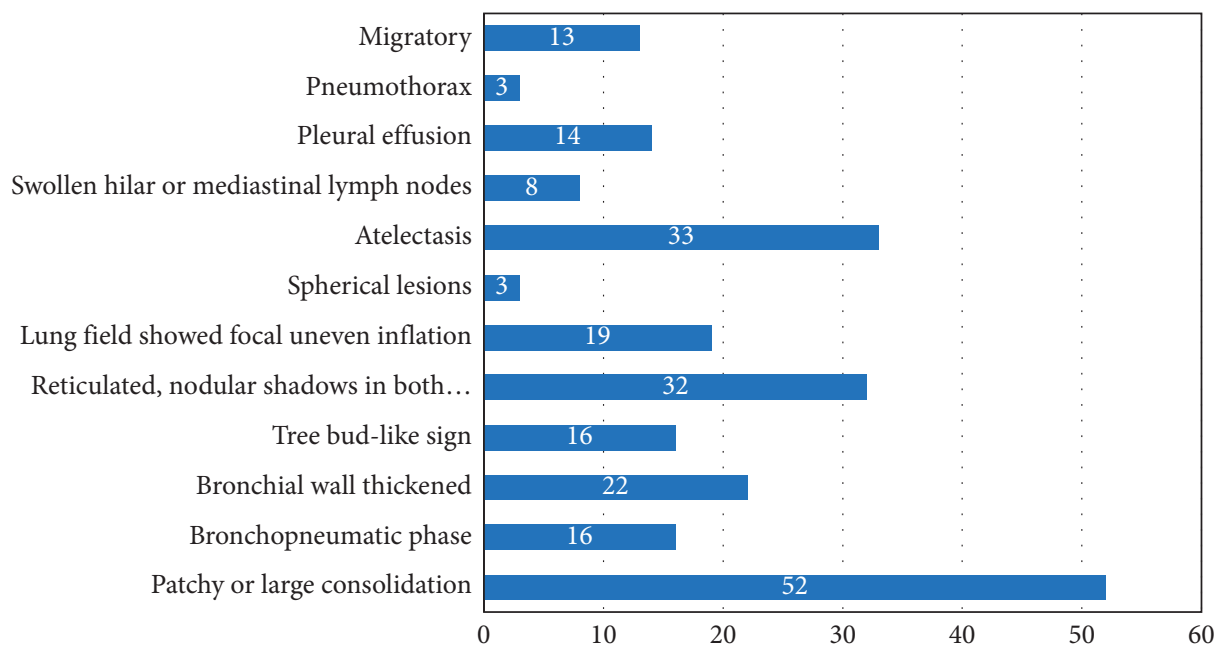

FIgURE 7: Analysis of imaging manifestations of children. 
3.5. Analysis of Fiberoptic Bronchoscopy and Cell Count of Bronchoalveolar Lavage Fluid in Children. In 69 cases of BAL children with fiberoptic bronchoscopy, swelling and congestion of the bronchial mucosa of the diseased site were seen under the microscope, viscous secretions were visible, and the secretion volume was different. The microscopic examination of the diseased site was consistent with the imaging changes. According to whether fiberoptic bronchoscopy BAL was performed, all children were divided into a lavage group (BAL group, $n=69$ ) and a nonlavage group (non-BAL group, $n=11$ ). The results obtained after counting the BALF of the two groups of children are shown in Table 1. The total number of cells and the proportion of neutrophils in the BALF of the BAL group were much higher than those of the non-BAL group $(P<0.05)$.

3.6. Comparison of Recovery Rate and Total Effective Rate between the Two Groups. After two weeks of treatment, the recovery rate and total effective rate of the two groups of children were analyzed and compared, as shown in Figure 8. There were 56 recovered cases $(81.16 \%)$ in the BAL group and 5 recovered cases $(45.45 \%)$ in the non-BAL group $(P<0.05)$. The total effective rate of the two groups of children was $100 \%$ both.

\section{Discussion}

At present, more and more attentions are paid to medical imaging image segmentation technology all over the world, which can find the location of the lesion in the early diagnosis accurately, thereby improving the cure rate and survival rate of patients greatly $[9,10]$. This study compared and analyzed the accuracy and denoising performance of the three edge operators (SO, PO, and RO) and the classic WSA and SIOA algorithms in the segmentation of CT medical images of children with RMPP. The results proved that the OA of the RO, PO, and SO was $0.935,0.925$, and 0.0.931, respectively, and the OA was 0.855 for WSA and 0.847 for SIOA. These data represented that the OAs of three edge algorithms were higher than those of the WSA and SIOA. OA refers to the ratio of the intersection and union between the divided area of the algorithm and the area manually drawn by the expert, which represents the accuracy of the algorithm. The higher the OA value, the higher the accuracy. The results revealed that the accuracies of edge algorithms in medical image segmentation were higher than those of WSA and SIOA. In addition, it was found that the FPR and FNR of the three edge algorithms were lower in contrast to those of the WSA and SIOA, indicating that the probability of FPR and FNR was lower, which indirectly proved that the accuracy of the edge algorithm was higher, which was consistent with previous studies [11].

In addition, the comparison results of different algorithms on lung CT image segmentation and denoising performance showed that the PSNR of RO, SO, PO, WSA, and SIOA was 27.916, 27.855, 27.796, 26.554, and 26.871, respectively. Thus, the PSNRs of the three edge algorithms were higher than those of the WSA and SIOA. PSNR is an
TABLE 1: Analysis of cell count in bronchoalveolar lavage fluid in two groups of children

\begin{tabular}{lcc}
\hline Indicator & BAL group & Non-BAL group \\
\hline Total number of cells $\left(\times 10^{6} / \mathrm{mL}\right)$ & $1.63 \pm 0.31^{*}$ & $0.59 \pm 0.08$ \\
Macrophages (\%) & $86.29 \pm 4.62$ & $91.05 \pm 5.18$ \\
Lymphocyte (\%) & $3.64 \pm 0.79$ & $4.11 \pm 0.84$ \\
Neutrophils (\%) & $11.37 \pm 1.22^{*}$ & $1.97 \pm 1.45$ \\
Eosinophils (\%) & $0.68 \pm 0.07$ & $0.35 \pm 0.06$ \\
\hline
\end{tabular}

Note: *indicated $P<0.05$ in contrast to the non-BAL group.

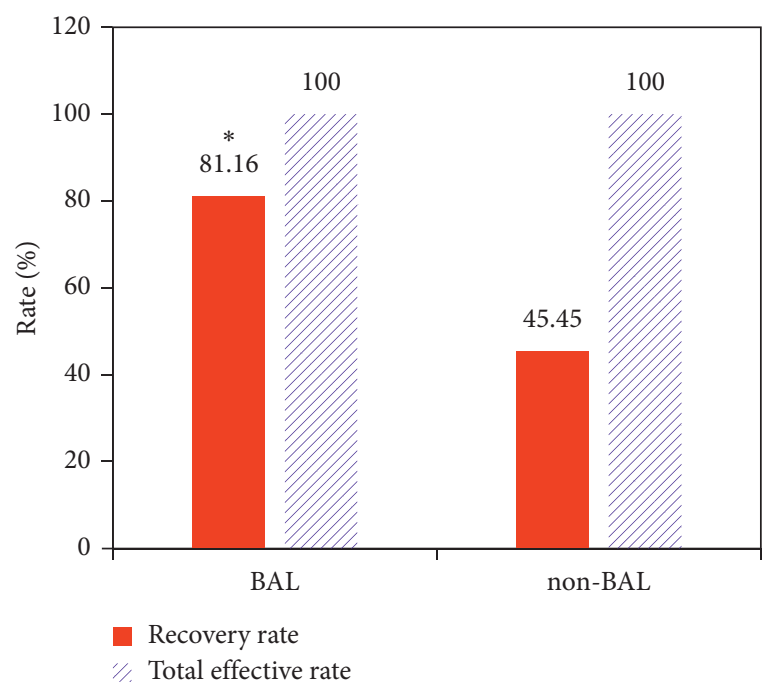

Figure 8: The recovery rate and total effective rate of the two groups of children. Note: * suggests $P<0.05$ in contrast to the nonBAL group.

objective evaluation standard to measure the denoising performance. The larger the value, the higher the denoising performance. It proved that the edge algorithm was better than the WSA and SIOA in denoising performance, which was consistent with previous research [12]. In addition, the $\mathrm{E}$ value of the WSA was the highest, followed by the SO; the E values of the RO, PO, and SIOA were close to each other. The $E$ value indicates the strength of the vertical or horizontal edge retention of the image after denoising. The larger the $\mathrm{E}$ value, the better the retention of gradient information and the stronger the retention of the edge. The analysis based on the $\mathrm{E}$ value showed that the edge retention of the WSA was stronger, and the edge retentions of the three edge algorithms were slightly lower than that of the WSA, which was similar to research results of Sousa et al. [13].

There is no early specific diagnosis method for RMPP, which is currently diagnosed with clinical examination and imaging examination. MP is a special pathogenic microorganism between virus and bacteria. The pathogenesis of RMPP is not clear, and most studies believe that it is mainly caused by infectious inflammation caused by the local growth and reproduction of MP in lung tissue, and further damage is caused by systemic and local immune responses $[14,15]$. MP mainly infects the epithelial cells from the respiratory tract to the respiratory bronchioles, causing the children to develop fever, cough, and other symptoms [16], 
which is consistent with the results of this study. The results disclosed that there were 47 cases $(58.75 \%)$ with fever $>39.0^{\circ} \mathrm{C}$, 58 children $(72.5 \%)$ had the fever duration of $7-14$ days, and all children had cough symptoms. Thus, children with RMPP mainly suffered from high fever and irritating dry, most of which had a fever duration of 7-14 days and fewer early signs, which was consistent with the research of $\mathrm{Qu}$ [17]. The chest CT imaging findings of children with RMPP were mostly patchy or large-scale consolidation, reticular or nodular shadows of both lungs, and atelectasis, which was consistent with study of Chen et al. [18]. In addition, the bronchial wall thickening and small tree bud sign were considered to be important imaging changes in adult infection with MP, so special attention should be paid during the clinical examination.

Fiberoptic bronchoscopy is an important tool for clinical diagnosis of RMPP. This study found that 69 cases of fiberoptic bronchoscopy in BAL children showed swelling and congestion of the bronchial mucosa at the lesion site, visible viscous secretions, and varying amounts of secretion. The imaging changes were consistent and related to the pathological changes caused by MP. Most of them were caused by the secretion of viscous secretions, resulting in local bronchial ventilating [19]. In addition, the BALF of BAL group and the non-BAL group were analyzed by cytological counting. The results indicated that the total number of cells and the proportion of neutrophils in the BALF of the BAL group were increased greatly than that in the non-BAL group. Related data suggested that MP infection could cause local aggregation and activation of neutrophils, indicating that neutrophils played a reliable role in the occurrence and development of RMPP, which was consistent with the research of Shimizu [20] and Li et al. [21]. Studies have shown that MP can temporarily inhibit the function of T lymphocytes and clear CD4+ T lymphocytes, leading to short-term immunosuppression, which may be the cause of the increase of neutrophils caused by MP infection, resulting in the local inflammation [22], but the specific mechanism of action had to be further studied. After 2 weeks of treatment for the two groups of children, 56 cases $(81.16 \%)$ in the BAL group were cured, and 5 cases $(45.45 \%)$ in the non-BAL group were cured with statistical meaningfulness $(P<0.05)$. The total effective rate of the two groups of children was $100 \%$ both. These results indicated that fiberoptic bronchoscopy lavage had a reliable therapeutic effect on RMPP, which may be because that the lavage treatment could effectively remove the lower respiratory tract viscous secretions and harmful pathogenic microorganisms, so as to alleviate airway obstruction, thereby improving the treatment effect of children with RMPP.

\section{Conclusion}

There were some limitations for this study. For example, it did not thoroughly analyze the recovery effect of BAL on the clinical characteristics and imaging manifestations of children with RMPP. This was because the children had been discharged from the hospital, and the follow-up review of most children was difficult to be realized. In the future, a following study would focus on these limitations to collect the clinical characteristics and imaging data of the children during the review and use the telephone to check the children's recovery status. The results of this study showed that the ECA had better accuracy and denoising performance in lung CT image segmentation. The clinical characteristics, imaging characteristics, and cytological components of children with RMPP had been changed, and BAL had a good therapeutic effect on it. In conclusion, this study could provide a positive effect for the early diagnosis and treatment of RMPP.

\section{Data Availability}

No data were used to support this study.

\section{Conflicts of Interest}

The authors declare that they have no conflicts of interest.

\section{References}

[1] H. Lee, K. W. Yun, H. J. Lee, and E. H. Choi, “Antimicrobial therapy of macrolide-resistantMycoplasma pneumoniaepneumonia in children," Expert Review of Anti-infective Therapy, vol. 16, no. 1, pp. 23-34, 2018.

[2] S. Kumar, "Mycoplasma pneumoniae: a significant but underrated pathogen in paediatric community-acquired lower respiratory tract infections," Indian Journal of Medical Research, vol. 147, no. 1, pp. 23-31, 2018.

[3] S. Kumar, R. D. Roy, G. Sethi, and S. R. Saigal, "Mycoplasma pneumoniae infection and asthma in children," Tropical Doctor, vol. 49, no. 2, pp. 117-119, 2019.

[4] J. C. Silva, P. Braga, D. Coutinho, and C. Fernades, "Refractory tracheoesophageal fistula management with amplatzer occluder (R) placement," Revista Española de Enfermedades Digestivas, vol. 112, no. 9, pp. 733-734, 2020.

[5] Y. Yang, D. Tian, and B. Wu, "A fast and reliable noise-resistant medical image segmentation and bias field correction model," Magnetic Resonance Imaging, vol. 54, pp. 15-31, 2018.

[6] L. Gui, C. Li, and X. Yang, "Medical image segmentation based on level set and isoperimetric constraint," Physica Medica, vol. 42, pp. 162-173, 2017.

[7] E. R. Arce-Santana, A. R. Mejia-Rodriguez, E. Martinez-Peña et al., "A new Probabilistic Active Contour region-based method for multiclass medical image segmentation," Medical, \& Biological Engineering \& Computing, vol. 57, no. 3, pp. 565-576, 2019.

[8] S. M. Plaza and J. Funke, "Analyzing image segmentation for connectomics," Frontiers in Neural Circuits, vol. 12, p. 102, 2018.

[9] A. Khadidos, V. Sanchez, and C.-T. Li, "Weighted level set evolution based on local edge features for medical image segmentation," IEEE Transactions on Image Processing, vol. 26, no. 4, pp. 1979-1991, 2017.

[10] G. Wang, J. Xu, Q. Dong, and Z Pan, “Active contour model coupling with higher order diffusion for medical image segmentation," International Journal of Biomedical Imaging, vol. 2014, Article ID 237648, 8 pages, 2014.

[11] K. Suzuki, H. Saji, K. Aokage et al., "Comparison of pulmonary segmentectomy and lobectomy: safety results of a randomized trial," The Journal of Thoracic and Cardiovascular Surgery, vol. 158, no. 3, pp. 895-907, 2019. 
[12] W. Cheng, L. Ma, T. Yang, J. Liang, and Y. Zhang, "Joint lung CT image segmentation: a hierarchical bayesian approach," PloS One, vol. 11, no. 9, Article ID e0162211, 2016.

[13] A. M. Sousa, S. B. Martins, A. X. Falcão, F. Reis, E. Bagatin, and K. Irion, "ALTIS: a fast and automatic lung and trachea CT-image segmentation method," Medical Physics, vol. 46, no. 11, pp. 4970-4982, 2019.

[14] P. M. Meyer Sauteur, W. W. J. Unger, A. M. C. van Rossum, and C. Berger, "The art and science of diagnosing mycoplasma pneumoniae infection," The Pediatric Infectious Disease Journal, vol. 37, no. 11, pp. 1192-1195, 2018.

[15] T. Shimizu, "Pathogenic factors of mycoplasma," Nippon Saikingaku Zasshi, vol. 70, no. 4, pp. 369-374, 2015.

[16] R. Ranjbar and M. Halaji, "Epidemiology of Mycoplasma pneumoniae prevalence in Iranian patients: a systematic review and meta-analysis," Journal of Medical Microbiology, vol. 68, no. 11, pp. 1614-1621, 2019.

[17] J. Qu, S. Chen, F. Bao, L. Gu, and B. Cao, "Molecular characterization and analysis of Mycoplasma pneumoniae among patients of all ages with community-acquired pneumonia during an epidemic in China," International Journal of Infectious Diseases, vol. 83, pp. 26-31, 2019.

[18] L. Chen, J. Liu, S. Zhao, Y. Yang, and J. Wu, "Clinical features and treatment of refractory Mycoplasma pneumoniae pneumonia unresponded to conventional dose methylprednisolone in children," Zhonghua Er Ke Za Zhi, vol. 52, no. 3, pp. 172-176, 2014.

[19] H. Zhang, Z. Chen, and H. Liu, "Pathogenic mechanism of Mycoplasma pneumoniae infections," Zhonghua $\mathrm{Er} \mathrm{Ke} \mathrm{Za}$ Zhi, vol. 54, no. 2, pp. 94-97, 2016.

[20] T. Shimizu, "Inflammation-inducing factors of mycoplasma pneumoniae," Frontiers in Microbiology, vol. 7, p. 414, 2016.

[21] W. Li, C. Ban, J. Zhang, Y Hu, B Han, and B Han, "Correlation study of cough variant asthma and mycoplasma pneumonia infection in children," Pakistan Journal of Pharmaceutical Sciences, vol. 30, no. 3, pp. 1099-1102, 2017.

[22] N. Li, Y. P. Mu, J. Chen, and B. Li, "Value of absolute counts of lymphocyte subsets in the early prediction of refractory Mycoplasma pneumoniae pneumonia in children," Zhong Guo Dang Dai Er Ke Za Zhi, vol. 21, no. 6, pp. 511-516, 2019. 